\title{
Visual Behavior Modeling of Hazard Identification Assessment From Eye-Tracking Data
}

\author{
Abner Cardoso da Silva ${ }^{1,2}$, Alberto Barbosa Raposo ${ }^{1,2}$ and Cesar Augusto Sierra Franco ${ }^{1,2}$ \\ acardoso@tecgraf.puc-rio.br, abraposo@tecgraf.puc-rio.br, casfranco@tecgraf.puc-rio.br \\ ${ }^{1}$ Department of Informatics, ${ }^{2}$ Tecgraf Institute
}

\begin{abstract}
The easier access to virtual reality head-mounted displays have assisted the use of this technology on research. In parallel, the integration of those devices with eye-trackers enabled new perspectives of visual attention analysis in virtual environments. Different research and application fields found in such technologies a viable way to train and assess individuals by reproducing, with low cost, situations that are not so easily recreated in real life. In this context, our proposal aims to develop a model to measure characteristics of safety professional's gaze behavior during the hazard detection process.
\end{abstract}

\section{INTRODUCTION}

Due to its popularization and price reduction, virtual reality (VR) head-mounted displays (HMD) have been largely disseminated. This allowed the use of those devices in different contexts, from entertainment to more serious uses like simulations of real world situations [1]. In the industry, simulations have received a great amount of attention, since it allows the creation of controlled and immersive applications, making easier to reproduce uncommon and expensive real life situations, besides, it enables the extraction of different types of data that may not be as easy in real life. This allows a more detailed analysis of the user's behavior in such environments.

The image presented to the user in this kind of media is strongly tied to the its point of view, in other words its head and eye orientation. In this context, seeing potential benefits to rendering and user interaction, companies like HTC have integrated eye tracking devices to its more recent HDMs. The data collected by such devices can be assessed to better understand a user's distribution of visual attention when navigating in a virtual environment, as well as to evaluate which aspects of the environment are more attractive to the user.

The area of hazard detection in work environments have received great contributions from those technologies. The creation of immersive and realistic virtual environments allow the assessment and training of safety professionals without exposing them to risks, besides, it reduces the costs associated with the process [2]. Furthermore, the analysis of eye tracking data and the metrics extracted from them, makes possible to better evaluate a participant performance in a given test. Such tools are commonly composed of a virtual environment that simulates real situations, where participants must indicate the existence of risks to life and material goods. To indicate the risks, the participant uses a controller interface (e.g. motion controllers).

Our work proposes the creation of a model to quantify characteristics of safety professionals' gaze behavior during the process of training or assessment in hazard identification, such aspects as doubt, tiredness, hastiness, etc. The model will take into account both the user gaze data provided by the eye tracker, and the controller position and orientation in the virtual world. We expect that our work will give better insights of user behavior in hazard detection activities, and contribute to better evaluate safety professionals' abilities.

\section{Motivation}

The training and assessment of safety professionals may be expensive and dangerous, as it is necessary to reproduce real situations with real risks. Furthermore, the performance of an unprepared professional may result in serious consequences to life or material goods. Therefore, a model capable of better evaluate this process, thus reducing the costs associated with it, is of great importance to the area.

\section{RELATED WORK}

The feasibility of VR simulations with HMD is assessed in the study of Pai et al. [3], where the authors evaluate the hazard anticipation of drivers of different ages by analyzing eye-tracking data. It is reported that VR headsets are a viable way to measure a participant's performance, since it has lower costs than traditional simulations and makes possible to accommodate multiple users in a single scene. This study focus on demonstrating the efficiency of VR simulations, so it did not provide a deeper evaluation of eye-tracking metrics, it analyses only raw gaze data.

Hasanzadeh, Esmaelli and Dodd [4] measure the importance of safety knowledge, hazard recognition and attention in order to prevent accidents in construction sites. The authors use a traditional eye-tracking setup to evaluate the visual search strategies of 27 construction workers. The results show that experience and previous exposure to accidents in the work environment have a significant impact in the way attention is allocated. This work aims to analyze eye-tracking measures, therefore it does not use VR, but propose its use as a way to improve the training methods to hazard detection in construction sites. 
Marwecki et al. [5] show the use of eye-tracking in virtual reality as a way to identify the focus of attention in order to change part of the scene without the user noticing. To know when to change the scene, the authors created a model that represents the user's attention, spatial memory and intention.

\section{Proposed Solution}

Our work proposes the development of a model to evaluate the hazard detection ability of safety professionals. Data will be collected through a virtual reality environment that presents real life hazardous situations. In this system, the user will be positioned in the center of a sphere mapped with equirectangular images of real locations with objects or situations that may represent some kind of risk to the work environment. The user's objective is to efficiently identify every hazard in the scenario within a time limit. The user uses a wireless motion controller to accurately indicate the location of hazards in the image.

Based on the feedback of safety specialists, we will develop a rule-based model to read the metrics extracted from the eyetacking data, and the orientation of the controller to produce concise measures that evaluate the user's efficiency during the evaluation process.

\section{Preliminary Results}

We made a literature review to identify relevant works that describe which eye-tracking metrics are commonly used to evaluate visual attention. The selected metrics were implemented in Python and evaluated with data collected in a virtual environment developed using the game engine Unity. To assist with the visualization and validation of the metrics, heatmaps and scanplots were implemented. Figures 1 and 2 show examples of both types of visualizations applied to data collected in a multiple-choice cognitive assessment task. With those visualization techniques it is possible to identify one's gaze trajectory and the most viewed locations in an image. The data collection application is already integrated with the HTC Vive ProEye and the HTC Vive wireless controller. The next step is to review the literature to find methods of rule-based systems and adapt it to this work's context.

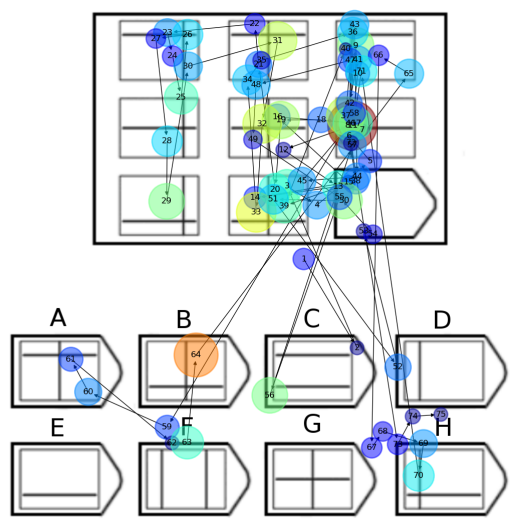

Fig. 1. Example of a scanplot visualization for a cognitive assessment task.
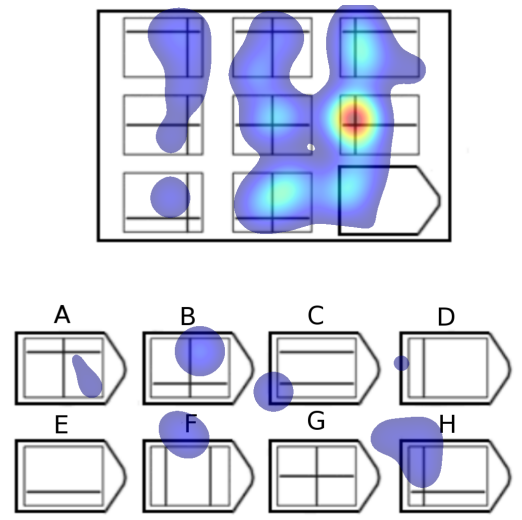

Fig. 2. Example of a heatmap visualization for a cognitive assessment task.

\section{CONCLUSION}

The reproduction of dangerous situations to assess safety professionals' decision making and attention requires high financial costs. Virtual reality with head-mounted displays is proposed as a low cost alternative to those types of assessments, since it allows the creation of a controlled and immersive virtual environment. Those devices make possible the collection and processing of multiple types of user data, granting a better understanding of user's behaviors and exploration patterns. Therefore, our work proposes the creation of a rule-based system to characterize visual attention during hazard detection tasks in virtual reality, using gaze data and the user interaction with a motion controller as inputs. Based on literature review we implemented eye-tracking metrics and a VR application for data collection. As next steps we aim to review the literature to better understand and adapt rule-based models to this work's scope.

\section{REFERENCES}

[1] S. J. Anbro, A. J. Szarko, R. A. Houmanfar, A. M. Maraccini, L. H Crosswell, F. C. Harris, M. Rebaleati, and L. Starmer, "Using virtual simulations to assess situational awareness and communication in medical and nursing education: A technical feasibility study," Journal of Organizational Behavior Management, pp. 1-11, 2020.

[2] E. Isleyen and H. Duzgun, "Use of virtual reality in underground roof fall hazard assessment and risk mitigation," International Journal of Mining Science and Technology, vol. 29, no. 4, pp. 603-607, 2019.

[3] G. Pai Mangalore, Y. Ebadi, S. Samuel, M. A. Knodler, and D. L. Fisher, "The promise of virtual reality headsets: Can they be used to measure accurately drivers' hazard anticipation performance?" Transportation research record, vol. 2673, no. 10, pp. 455-464, 2019.

[4] S. Hasanzadeh, B. Esmaeili, and M. D. Dodd, "Measuring the impacts of safety knowledge on construction workers' attentional allocation and hazard detection using remote eye-tracking technology," Journal of management in engineering, vol. 33, no. 5, p. 04017024, 2017.

[5] S. Marwecki, A. D. Wilson, E. Ofek, M. Gonzalez Franco, and C. Holz, "Mise-unseen: Using eye tracking to hide virtual reality scene changes in plain sight," in Proceedings of the 32nd Annual ACM Symposium on User Interface Software and Technology, 2019, pp. 777-789. 